Seventh International Conference on

Geometry, Integrability and Quantization

June 2-10, 2005, Varna, Bulgaria

IvaÃŕlo M. Mladenov and Manuel de León, Editors

SOFTEX, Sofia 2005, pp 1-20

\title{
EXTERIOR DIFFERENTIAL SYSTEMS AND BILLIARDS
}

\section{J.M. LANDSBERG}

Department of Mathematics, Texas A\& M University, Mailstop 3368, College Station, TX 77843-3368, USA

\begin{abstract}
I describe work in progress with Baryshnikov and Zharnitsky on periodic billiard orbits that leads one to an exterior differential system (EDS). I then give a brief introduction to EDS illustrated by several examples.
\end{abstract}

\section{Introduction}

The purpose of these notes is to introduce the reader to the techniques of exterior differential systems (EDS) in the context of a problem in billiards. The approach in this article is different from that of [13] and [16], which begin with a study of linear Pfaffian systems, an important special class of EDS. The billiard problem results in an EDS that is not a linear Pfaffian system, so these notes deal immediately general EDS. For the interested reader, two references regarding EDS are [5] and [13]. The first is a definitive reference and the second contains an introduction to the subject via differential geometry. For more details about anything regarding EDS the reader can consult either of these two sources. Cartan's book on EDS [10] is still worth looking at, especially the second half, which is a series of beautiful examples.

We generally will work in the real analytic category, although all the non-existence results discussed here imply non-existence of smooth solutions.

\section{Notation}

If $M$ is a differentiable manifold we let $T M, T^{*} M$ denote its tangent and cotangent bundles, $\Omega^{d}(M)$ the set of differential forms on $M$ of degree $d$ and $\Omega^{*}(M)=$ $\oplus_{d} \Omega^{d}(M)$. If $I \subset T^{*} M$ is a subbundle (more precisely, subsheaf), then we let $\{I\}_{\text {diff }} \subset \Omega^{*}(M)$ denote the differential ideal generated by $I$, i.e, all elements of $\Omega^{*}(M)$ of the form $\alpha \wedge \phi+d \beta \wedge \psi$ where $\alpha, \beta \in I$ and $\phi, \psi \in \Omega^{*}(M) .\left\{v_{1}, \ldots, v_{n}\right\}$ denotes the linear span of the vectors $v_{i}$ if they are vectors, and the subbundle of $\Omega^{1}(M)$ they generate if they are one-forms. 


\section{Origin of the billiard problem}

Let $D \subset \mathbb{R}^{2}$ be a convex domain with its flat metric. Let $\Delta$ denote the standard Laplacian on $D$. Then Weyl [19] conjectured and Ivrii [14] proved

$$
\left\{\text { number of eigenvalues of } \Delta \leq \lambda^{2}\right\}=\frac{1}{\pi} \operatorname{area}(D) \lambda^{2} \pm \frac{1}{4} \operatorname{length}(\partial D) \lambda+o(\lambda)
$$

where more precisely, Weyl proved the first term is indeed the leading term and Ivrii proved the correction term (+ with Dirchlet, - with Neumann boundary conditions), but subject to the following possibly extraneous hypothesis:

That there does not exist a two parameter family of periodic billiard trajectories in $D$.

In fact Weyl and Ivrii work in $n$ dimensions but we have restricted to $n=2$ for notational simplicity. Also, Ivrii's actual restriction was that there was not a set of positive measure of periodic billiard trajectories in the space of all trajectories, but for the problem at hand, that is equivalent to the statement above, as remarked in [17].

I will report on joint work with Y. Barishnikov and V. Zharnitsky investigating whether this additional hypothesis is actually necessary or not. But first, I must explain the hypothesis.

\section{Billiards}

Let $C \subset \mathbb{R}^{2}$ be a smooth curve. A billiard trajectory is defined by a particle traveling in straight lines in the interior of $C$ and reflecting at the boundary subject to the law that the angle of incidence with the tangent line to the curve equals the angle of reflection.

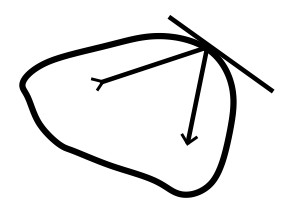

Figure 1.

A trajectory is periodic if it closes up and repeats itself. The number of collisions it has with the boundary of $C$ before repeating is called its period.

For example, if $C$ is a circle, then there are many periodic trajectories.

Moreover, given a periodic trajectory in the circle one can construct a one parameter family of such by varying the initial point and keeping the angle constant. It is also true that given an ellipse and a periodic trajectory on it, one can still obtain 


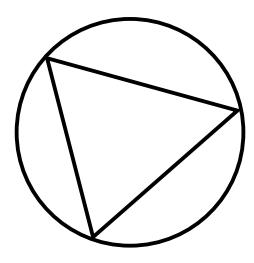

Figure 2.

a one parameter family of periodic trajectories if one moves the angle just right as one displaces the initial point.

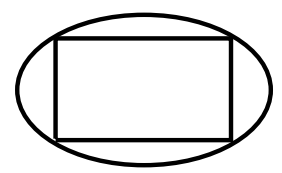

Figure 3.

One can locally parametrize the space of trajectories by putting a local parameter on the curve (e.g. arclength) and measuring the angle of the trajectory with the tangent line to the curve (one thinks of shooting out a trajectory from that point). In particular, the set of trajectories is a two dimensional space and the existence of a two parameter family of periodic trajectories would mean that there is some point on the curve $C$ such that no matter what small perturbation of the initial angle and initial point one makes, the resulting trajectory is still periodic.

\section{Sound preposterous?}

Ivrii thought so. In fact, legend has it that Ivrii was attempting to prove the correction term to Weyl's formula and realized it he could prove it under the assumption that there are no periodic billiard trajectories in the domain. Fortunately for him (he thought), he was at Moscow State University, where there were many world experts on billiards. Allegedly he went in to ask them if there could be such a curve - they quickly answered: "Of course not!", so he said "Great! may I please have a proof?"- they said certainly. They had trouble coming up with a full argument immediately so they told him to come back later in the afternoon. He returned later that afternoon and they told him that perhaps it would be better to return the next day... then it became the next week, ... All this was nearly 30 years ago and the question is pretty much as open today as it was then.

Some things are known: for a similar problem the answer is that there are such things: there exist compact surfaces with Riemannian metrics, all of whose geodesics 
are closed [11]. These are called Zoll surfaces and there are more of them than was originally expected.

The progress on Ivrii's question is as follows: we may break it up into a series of questions based on the period of the trajectory. It is easy to see that there can be at most a 1-parameter family of two-periodic trajectories. (Hint: what happens when you change the angle a little?)

In 1989 Rychlik [17] proved that there are no curves supporting an open set of 3-periodic trajectories. Now there are three published proofs of Rychlik's theorem $[17,18,20]$, and in these lectures I will give you a fourth. The four periodic case is still open and that is the subject of my current research with Baryshnikov and Zharnitsky.

\section{Setting up the problem}

(The results in this section are joint work with Baryshnikov and Zharnitsky.) The problem is local. If we want an $n$-periodic trajectory, we only need $n$ bits of curve. We can later close up the bits any way we please (as long as it closes convexly).

Let $z_{1}, \ldots, z_{n} \in \mathbb{R}^{2}$. We want to construct $n$ (germs of) curves, one passing through each point. The initial points determine an initial $n$-gon which in turn tells us what the tangent lines to the curves must be at the $z_{i}$. I.e., the $n$ points immediately determine the zero-th and first order terms of the Taylor series for the curves.

Let

$$
N_{i}=\frac{z_{i}-z_{i+1}}{\left|z_{i}-z_{i+1}\right|}-\frac{z_{i}-z_{i-1}}{\left|z_{i}-z_{i-1}\right|}
$$

and note that $N_{i}$ points in the direction of the tangent line to the curve we are trying to construct. Let $n_{i}=N_{i} /\left|N_{i}\right|$. Let $J n_{i}$ denote the rotation counterclockwise of $n_{i}$ by $\pi / 2$. We have the following picture

The tangent line at $z_{i}$ must be perpendicular to $J n_{i}$. Let $\Sigma=\left(\mathbb{R}^{2}\right)^{\times n}$ denote the naïve configuration space (the actual space is an open subset of this) where $p=\left(z_{1}, \ldots, z_{n}\right) \in \Sigma$ is our initial point. Define

$$
\psi_{i}:=\left\langle J n_{i}, d z_{i}\right\rangle \in \Omega^{1}(\Sigma)
$$

and for future reference set $\eta^{i}=\left\langle n_{i}, d z_{i}\right\rangle$, let $\alpha_{i}$ be the angle between $e_{i-1}$ and $J n_{i}$, and let $l_{i}$ be the length of the section from $z_{i}$ to $z_{i+1}$.

We have a distribution $\Delta$ on $\Sigma$, namely

$$
\Delta=\operatorname{ker}\left\{\psi_{1}, \ldots, \psi_{n}\right\} \subset T \Sigma
$$

Any two parameter family of $n$-periodic trajectories corresponds to an immersed surface $M^{2} \hookrightarrow \Sigma$ which is everywhere tangent to $\Delta$ and subject to some additional genericity conditions. More precisely, we have 


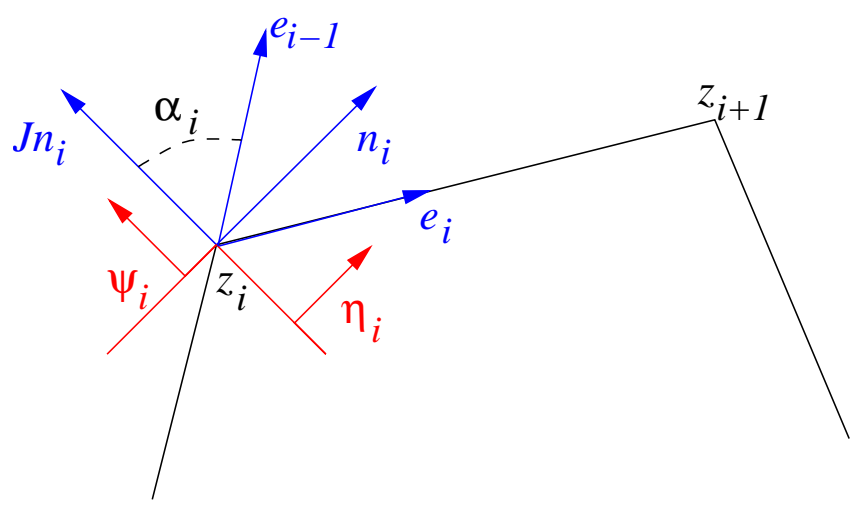

Figure 4.

Proposition 1. [7] There exists a one to one correspondence between (segments of) curves admitting an open subset of n-periodic trajectories and immersed surfaces $i: M^{2} \rightarrow \Sigma$ tangent to $\Delta$ satisfying

1. no two points coincide

2. no three points are colinear

3. Any two consecutive points "move independently" in the manner made precise in condition (1) described below.

Note that the first two conditions are zero-th order conditions regarding the initial point in $\Sigma$ about which we want to construct the surface. The last is a first order condition which may be described as follows:

Note that $\left(\eta_{p}^{1}, \ldots, \eta_{p}^{n}, \psi_{p}^{1}, \ldots, \psi_{p}^{n}\right)$ gives a basis of $T_{p}^{*} \Sigma$ and that this basis varies smoothly - one says $\left(\eta^{i}, \psi^{i}\right)$ form a coframing of of $\Sigma$. The precise form of condition (3) for proposition 1 is that

$$
i^{*}\left(\eta^{i} \wedge \eta^{i+1}\right) \text { is nonvanishing } \forall 1 \leq i \leq n
$$

where we use the convention that for indices $n+1=1$.

How can we determine the existence of such surfaces?

Were we looking for $n$-folds, the answer would be given by the Frobenius theorem:

Theorem 1 (Frobenius theorem). Given pointwise linearly independent one-forms $\psi^{1}, \ldots, \psi^{n}$ on a manifold $X^{m}$, there exists an immersed submanifold $i: M^{m-n} \rightarrow X$ passing through $p \in X$ on which $i^{*}\left(\psi^{j}\right) \equiv 0$ for all $j$ (i.e., with $T_{x} M=\Delta_{x}:=$ $\operatorname{ker}\left\{\psi_{j}\right\}$ for all $\left.x \in M\right)$ if in a neighborhood of $p$ there exist one-forms $\alpha_{j}^{i} \in$ $\Omega^{1}(X)$ such that

$$
d \psi^{i}=\alpha_{1}^{i} \wedge \psi^{1}+\cdots+\alpha_{n}^{i} \wedge \psi^{n} \forall i
$$


The condition (2) is often expressed as $d \psi^{i} \equiv 0 \bmod \left\{\psi^{1}, \ldots, \psi^{n}\right\}$. In fact the individual forms don't matter, just their span, so we could write $I=\left\{\psi^{1}, \ldots, \psi^{n}\right\}$ and

$$
d \psi^{i} \equiv 0 \bmod I \forall i
$$

Another way to express it is that locally if, $X, Y$ are vector fields lying in $\Delta$, that $[X, Y]$ also lies in $\Delta$. (Exercise: verify that this is indeed equivalent.) Note that all these conditions involve beginning with first order information and differentiating it once - if everything is OK, then we are guaranteed solutions. That is, we can stop working after taking two derivatives.

Were we in the situation that there was just a single one-form, then Pfaff's theorem (see, e.g., [5] §1.3) guarantees existence of submanifolds of dimension roughly half the dimension of the manifold. Moreover, by computing the exterior derivative of the one-form one can determine the precise maximal dimension of a submanifold on which the form pulls back to be zero.

To deal with the general setting of determining existence of submanifolds on which an ideal of differential forms pulls back to be zero, an explicit algorithm was developed by Cartan and others. The algorithm also gives a rough estimate of the size of the space of such manifolds. (E.g., in the Frobenius theorem, there is a unique such manifold through a point but for Pfaff's theorem, there will be "functions" worth of solution manifolds through a point.)

The essential question is: Given a candidate tangent space (a first order admissible Taylor series), can we extend it? - i.e., can we "fit together" potential tangent spaces to obtain a solution submanifold?

\section{EDS terminology}

Let $V$ be a vector space, let $G(k, V)$ denote the Grassmannian of $k$-planes through the origin in $V$.

Definition 1. Let $\Sigma$ be a manifold Let $\mathcal{I} \subset \Omega^{*}(\Sigma)$ be a differential ideal, which we will call an exterior differential system. We let $\mathcal{I}_{j} \subset \Omega^{j}(\Sigma)$ denote the component in degree $j$ and we will henceforth assume $\mathcal{I}_{0}=\emptyset$. An integral manifold of $\mathcal{I}$ is an immersed submanifold $i: M \rightarrow \Sigma$ such that $i^{*}(\phi)=0$ for all $\phi \in \mathcal{I}$.

As with many things in mathematics, we will work infinitesimally with the goal of linearizing the problem of determing the integral manifolds of an EDS.

Definition 2. For $x \in \Sigma$, we let

$$
\mathcal{V}_{k}(\mathcal{I})_{x}:=\left\{E \in G\left(k, T_{x} \Sigma\right)|\phi|_{E}=0 \forall \phi \in \mathcal{I}\right\}
$$

which is called the variety of $k$-dimensional integral elements to $\mathcal{I}$ at $x$. We let $\mathbf{G}(k, T \Sigma)$ denote the Grassmann bundle, i.e., the bundle over $\Sigma$ whose fiber 
over $x \in \Sigma$ is $G\left(k, T_{x} \Sigma\right)$, and let $\mathcal{V}_{k}(\mathcal{I}) \subset \mathbf{G}(k, T \Sigma)$ denote the set of all $k$ dimensional integral elements.

The first step in the Cartan algorithm is Cartan's test: one compares a crude estimate (obtained from linear algebra calculations) of $\operatorname{dim} \mathcal{V}_{k}(\mathcal{I})$ with its actual dimension. If the two numbers agree, then the Cartan-Kähler theorem guarantees local existence of integral manifolds. We can think of it as saying "if the second order terms for the Taylor series look good, everything is good". If the test fails, we must take more derivatives to determine existence. This process is called prolongation. The Kuranishi prolongation theorem says that in principle one only needs to prolong a finite number of times before getting a definitive answer, but this is of little use in practice as the theorem gives no indication of how many times one must prolong (how many derivatives one needs to take). Before going into details, let's examine some examples to develop our intuition.

\section{PDE and EDS}

Example 1. Consider the PDE system for $u(x, y)$ given by

$$
\begin{aligned}
& u_{x}=A(x, y, u), \\
& u_{y}=B(x, y, u),
\end{aligned}
$$

where $A, B$ are given smooth functions. Since (3) specifies both partial derivatives of $u$, at any given point $p=(x, y, u) \in \mathbb{R}^{3}$ the tangent plane to the graph of a solution passing through $p$ is uniquely determined.

Whether or not the plane is actually tangent to a solution to (3) depends on whether or not the equations (3) are "compatible" as differential equations. For smooth solutions to a system of PDE, compatibility conditions arise because mixed partials must commute, i.e., $\left(u_{x}\right)_{y}=\left(u_{y}\right)_{x}$. In our example,

$$
\begin{aligned}
& \left(u_{x}\right)_{y}=\frac{\partial}{\partial y} A(x, y, u)=A_{y}(x, y, u)+A_{u}(x, y, u) \frac{\partial u}{\partial y}=A_{y}+B A_{u}, \\
& \left(u_{y}\right)_{x}=B_{x}+A B_{u},
\end{aligned}
$$

so setting $\left(u_{x}\right)_{y}=\left(u_{y}\right)_{x}$ reveals a "hidden equation", the compatibility condition

$$
A_{y}+B A_{u}=B_{x}+A B_{u} .
$$

and the Frobenius condition is exactly the vanishing of this equation. To see this let

$$
\theta=d u-A(x, y, u) d x-B(x, y, u) d y .
$$

Exercise: show that (4) holds iff $d \theta \equiv 0 \bmod \theta$.

Here we have the EDS $\mathcal{I}=\{\theta\}_{\text {diff }}$ on $\Sigma=\mathbb{R}^{3}$ but since this EDS comes from a PDE, we have an additional condition that we want our integral manifolds to 
satisfy, namely that $x, y$ are independent variables on a solution. We encode this by setting $\Omega=d x \wedge d y$ and making the following definitions:

Definition 3. Let $\mathcal{I} \subset \Omega^{*}(\Sigma)$ be a differential ideal, and $\Omega \in \Omega^{n}(\Sigma)$. The pair $(\mathcal{I}, \Omega)$ is called an exterior differential system with independence condition. An integral manifold of $\mathcal{I}$ is an immersed submanifold $i: M \rightarrow \Sigma$ such that $i^{*}(\phi)=0$ for all $\phi \in \mathcal{I}$ and $i^{*}(\Omega)$ is nonvanishing. Note that we really only need $\Omega$ up to scale and modulo $\mathcal{I}$, so we sometimes refer to an independence condition as an equivalence class of $n$-forms (the equivalence is up to scale and modulo $\mathcal{I}$ ).

Remark 1. One can attempt to obtain solutions to the system (3) by solving a succession of Cauchy problems. For example fix $y=0$ and solve the ODE

$$
\frac{d \tilde{u}}{d x}=A(x, 0, \tilde{u}), \quad \tilde{u}(0)=u_{0} .
$$

After solving (5), hold $x$ fixed and solve the initial value problem

$$
\frac{d u}{d y}=B(x, y, u), \quad u(x, 0)=\tilde{u}(x) .
$$

This determines a function $u(x, y)$ on some neighborhood of $(0,0)$. The problem is that this function may not satisfy our original equation, and it also may depend on the path chosen. The function is independent of path chosen precisely if the Frobenius condition holds, and in that case it gives the right answer too.

In general, given a first-order system of $r$ equations for $s$ functions $u^{a}$ of $n$ variables, there exists a change of coordinates so that the system takes the form

$$
\begin{aligned}
& u_{x^{1}}^{1}=f_{1}^{1}(\bar{x}, \bar{u}), \\
& \vdots \\
& u_{x^{1}}^{r_{1}}=f_{r_{1}}^{1}(\bar{x}, \bar{u}), \\
& u_{x^{2}}^{1}=f_{1}^{2}\left(\bar{x}, \bar{u}, \bar{u}_{x^{1}}\right), \\
& \vdots \\
& u_{x^{2}}^{r_{2}}=f_{r_{2}}^{2}\left(\bar{x}, \bar{u}, \bar{u}_{x^{1}}\right), \\
& \vdots \\
& u_{x^{n}}^{1}=f_{1}^{n}\left(\bar{x}, \bar{u}, \bar{u}_{x^{1}}, \ldots, \bar{u}_{x^{n-1}}\right), \\
& \vdots \\
& u_{x^{n}}^{r_{n}}=f_{r_{n}}^{n}\left(\bar{x}, \bar{u}, \bar{u}_{x^{1}}, \ldots, \bar{u}_{x^{n-1}}\right),
\end{aligned}
$$

where $\bar{x}=\left(x^{1}, \ldots, x^{n}\right), \bar{u}=\left(u^{1}, \ldots, u^{s}\right), u_{x^{j}}^{a}=\frac{\partial u^{a}}{\partial x^{j}}, 1 \leq a \leq s, 1 \leq j \leq n$, and $r_{1} \leq r_{2} \leq \ldots \leq r_{n}=s$ with $r=r_{1}+\ldots+r_{n}$ (see [9]). 
We may be able to produce solutions of this system by solving a series of Cauchy problems. However, we need to check that equations are compatible, i.e., that mixed partials commute:

$$
\frac{\partial}{\partial x^{i}} f_{\sigma}^{j}=\frac{\partial}{\partial x^{j}} f_{\sigma}^{i}, \quad 1 \leq i, j \leq n, \forall \sigma .
$$

Although it would be impractical to change any given system of PDE into the above form, converting this system to an EDS will guide us naturally to the analog of the above form. We can then apply a straightforward test that signals when no further compatibility conditions need to be checked.

\section{Cartan's test}

Let $\mathcal{I}$ be an EDS on a manifold $\Sigma$. Let $p \in \Sigma$ be a general point and $(p, E) \in \mathcal{V}_{n}(\mathcal{I})$ be a general point of $\mathcal{V}_{n}(\mathcal{I})$. The required generality can be made precise, see $[13,5]$ but we suppress that in these lectures. Intuitively, we want $(p, E)$ to be "like" its neighbors in some small open set in $\mathcal{V}_{n}(\mathcal{I})$.

Remark 2. Note that since we are dealing with (analytic) varieties, i.e., zero sets of analytic functions, there can be components to $\mathcal{V}_{n}(\mathcal{I})_{p}$. "A general point" means a general point of a given component.

As mentioned above, the test we are after will compare a codimension estimate obtained by linear algebra calculations with the codimension of a variety.

Definition 4. Let $E \in \mathcal{V}_{j}(\mathcal{I})_{p}$ and let $e_{1}, \ldots, e_{j}$ be a basis of $E$. Define

$$
H(E):=\left\{v \in T_{p} \Sigma \mid \phi\left(v, e_{1}, \ldots, e_{j}\right)=0 \forall \phi \in \mathcal{I}_{j+1}\right\}
$$

the polar space of $E$.

Note that

1. $H(E)$ is well defined (i.e., independent of our choice of basis),

2. $E \subset H(E)$ and

3. determining $H(E)$ is a linear calculation.

The quotient $H(E) / E$ may be thought of as the space of possible enlargements of $E$ from a $p$-dimensional integral element to a $(p+1)$-dimensional integral element. We will actually need to calculate the dimensions of a series of polar spaces.

Let $E \in \mathcal{V}_{n}(\mathcal{I})$. Fix a generic flag $E_{1} \subset \cdots \subset E_{n-1} \subset E_{n}=E$ in $E$. Let $c_{j}=\operatorname{codim}\left(H\left(E_{j}\right), T_{p} \Sigma\right)$, and set $c_{0}=\operatorname{codim} \mathcal{V}_{1}(\mathcal{I})=\operatorname{dim} \mathcal{I}_{1}$. Note that if $\Sigma$ has components, then codim $\mathcal{V}_{1}(\mathcal{I})$ can depend on the component, and for $j>1$, $\mathcal{V}_{j}(\mathcal{I})$ may have components even if $\Sigma$ has just one component. Therefore we will write $\operatorname{codim}_{E_{j+1}}\left(\mathcal{V}_{j+1}(\mathcal{I}), \mathbf{G}(j+1, T \Sigma)\right)$ to eliminate any possible ambiguity when discussing the codimension of $\mathcal{V}_{j+1}(\mathcal{I})$ at $E_{j+1}$. 
We have the following estimate

\section{Proposition 2.}

$\operatorname{codim}_{E_{j+1}}\left(\mathcal{V}_{j+1}, \mathbf{G}(j+1, T \Sigma)\right) \geq \operatorname{codim}_{E_{j}}\left(\mathcal{V}_{j}, \mathbf{G}(j, T \Sigma)\right)+\operatorname{codim}_{T_{p} \Sigma} H\left(E_{j}\right)$.

The inequality is intuitively reasonable as the first term on the right represent the conditions to have a $j$-dimensional integral element and the second term represents the new conditions for enlarging it to a $(j+1)$-dimensional integral element. Equality holding should be interpreted as $\mathcal{V}_{j+1}$ being "as large as possible" at $E_{j+1}$. Adding up these inequalities, we obtain

\section{Proposition 3.}

$$
\operatorname{codim}_{E}\left(\mathcal{V}_{n}, \mathbf{G}(n, T \Sigma) \geq c_{0}+c_{1}+\cdots+c_{n-1} .\right.
$$

The Cartan-Kähler theorem states that when equality holds (assuming our genericity hypotheses about $p$ and $E$ ), there exists an $n$-dimensional integral manifold through $p$ with tangent space $M$. The test for equality holding in (7) is called Cartan's test. If an integral element passes Cartan's test, we get a bonus - a coarse estimate of the size of the moduli space of integral manifolds through $p$. Namely if we set $s_{k}=c_{k}-c_{k-1}$ and let $k_{0}$ be the largest integer such that $s_{k_{0}}$ is nonzero, then integral manifolds depend roughly on $s_{k_{0}}$ analytic functions of $k_{0}$ variables. In particular if the largest $k_{0}$ is 0 , then integral manifolds depend only on a choice of constants, as in the Frobenius theorem.

\section{Other possibilities.}

$\mathcal{V}_{n}(\mathcal{I})_{p}=\emptyset$.

More precisely, there exists a Zariski open subset of $\Sigma$ over which there are no $n$ dimensional integral elements. In this case it is necessary to restrict to the (analytic) subvariety $\Sigma^{\prime} \subset \Sigma$ over which there are $n$-dimensional integral elements and start over, working at general points of $\Sigma^{\prime}$. Note that $\Sigma^{\prime}$ may have several components and that one must perform the test on each component separately. If $\operatorname{dim} \Sigma^{\prime}<n$ we are done, there are no $n$-dimensional integral manifolds.

\section{Cartan's test fails.}

Intuitively, this means we have not differentiated enough to uncover all compatibility conditions and we must take more derivatives. It turns out that, rather than taking higher derivatives, it is notationally simpler to start over on a larger space where our old derivatives are replaced by independent variables. (This corresponds to the standard process of converting any system of PDE to a first order system by adding additional variables.) 
More precisely, forgetting about $\mathcal{I}$ for the moment, on $\pi: \mathbf{G}(n, T \Sigma) \rightarrow \Sigma$, consider the following tautological system: given $(p, E) \in \mathbf{G}(n, T \Sigma)$, we have $E^{\perp} \subset$ $T_{p}^{*} \Sigma$. Define

$$
I_{(p, E)}:=\pi^{*}\left(E^{\perp}\right) .
$$

For good measure we add the independence condition determined by $\Lambda^{n}\left(\pi^{*}\left(T^{*} \Sigma / I\right)\right)$. Integral manifolds of the tautological system $\left(\{I\}_{\text {diff }}, \Omega\right)$ with $[\Omega] \in \Lambda^{n}\left(\pi^{*}\left(T^{*} \Sigma / I\right)\right)$ are precisely the Gauss images of immersed $n$-dimensional submanifolds $f: M \rightarrow \Sigma$. Now let's return to our original $\operatorname{EDS} \mathcal{I}$ on $\Sigma$ :

Definition 5. The prolongation of $\mathcal{I}$ is the pullback of the tautological system on $\mathbf{G}(n, T \Sigma)$ to $\mathcal{V}_{n}(\mathcal{I}) \subset \mathbf{G}(n, T \Sigma)$.

One then starts over with $\Sigma$ replaced by $\mathcal{V}_{n}(\mathcal{I})$ and $\mathcal{I}$ replaced by the pullback of the tautological system. One then performs Cartan's test, if it fails, one prolongs again, etc... For more details, see [13], §5.5.

\section{First examples of Cartan's test}

Example 0: arbitrary maps $\mathbb{R}^{2} \rightarrow \mathbb{R}^{2}$.

Let the first $\mathbb{R}^{2}$ have coordinates $x^{1}, x^{2}$, the second coordinates $u^{1}, u^{2}$ and let $\Sigma=$ $J^{1}\left(\mathbb{R}^{2}, \mathbb{R}^{2}\right) \simeq \mathbb{R}^{8}$ with coordinates $\left(x^{i}, u^{j}, p_{j}^{i}\right), 1 \leq i, j \leq 2$.

Given a map $f: \mathbb{R}^{2} \rightarrow \mathbb{R}^{2}$, we define the lift of $f$ to $\Sigma$ to be the set of points

$$
\left(x^{1}, x^{2}, f^{1}(x), f^{2}(x),\left.\left.\frac{\partial f^{1}}{\partial x^{1}}\right|_{x} \frac{\partial f^{1}}{\partial x^{2}}\right|_{x},\left.\frac{\partial f^{2}}{\partial x^{1}}\right|_{x},\left.\frac{\partial f^{1}}{\partial x^{2}}\right|_{x}\right),
$$

which is a coordinate version of the Gauss map of an immersion. Let

$$
\begin{aligned}
& \theta^{1}=d u^{1}-p_{1}^{1} d x^{1}-p_{2}^{1} d x^{2} \\
& \theta^{2}=d u^{2}-p_{1}^{2} d x^{1}-p_{2}^{2} d x^{2}
\end{aligned}
$$

Introduce the independence condition $\Omega=d x^{1} \wedge d x^{2}$. Then integral manifolds of the system $\left(\left\{\theta^{1}, \theta^{2}\right\}_{\text {diff } f}, \Omega\right)$ are in one to one correspondence with lifts of maps $f: \mathbb{R}^{2} \rightarrow \mathbb{R}^{2}$.

The manifold $\Sigma=J^{1}\left(\mathbb{R}^{2}, \mathbb{R}^{2}\right)$ equipped with the system $\left(\left\{\theta^{1}, \theta^{2}\right\}_{\text {diff }}, \Omega\right)$ is called the space of one-jets of mappings $\mathbb{R}^{2} \rightarrow \mathbb{R}^{2}$.

Let's perform Cartan's test:

Determination of $c_{0}+c_{1}$.

$c_{0}=2$ because $\operatorname{dim} \mathcal{I}_{1}=2$. The equations on any line $\{v\}$ are explicitly $\theta^{1}(v)=$ $0, \theta^{2}(v)=0$. 
To find $c_{1}$, we need to take a generic $\{v\} \in \mathcal{V}_{1}$. Write

$$
v=a^{1} \frac{\partial}{\partial x^{1}}+a^{2} \frac{\partial}{\partial x^{2}}+b_{a}^{i} \frac{\partial}{\partial p_{i}^{a}}+e_{a} \frac{\partial}{\partial u^{a}}
$$

where here and throughout we use the summation convention that repeated indices are to be summed over. $\theta^{j}(v)=e_{j}-p_{1}^{j} a^{1}-p_{2}^{j} a^{2}$ so we may take

$$
v=a^{1} \frac{\partial}{\partial x^{1}}+a^{2} \frac{\partial}{\partial x^{1}}+b_{a}^{i} \frac{\partial}{\partial p_{i}^{a}}+\left(p_{1}^{a} a^{1}+p_{2}^{a} a^{2}\right) \frac{\partial}{\partial u^{a}}
$$

where $a^{j}, b_{a}^{i}$ are (general) constants.

To determine $c_{2}$, we must find $\mathcal{I}_{2}$. First there are $\alpha \wedge \theta^{1}, \alpha \wedge \theta^{2}$ where $\alpha$ is any one-form. We also have $d \theta^{j}=-d p_{1}^{j} \wedge d x^{1}-d p_{2}^{j} \wedge d x^{2}$. To determine a possible enlargement of $\{v\}$ we must calculate

$$
d \theta^{j}(v, \cdot)=b_{1}^{j} d x^{1}-a^{1} d p_{1}^{j}+b_{2}^{j} d x^{2}-a^{2} d p_{2}^{j}
$$

So any vector $w$ in $H^{1}(\{v\})$ must satisfy the four linear equations

$$
\theta^{j}(w)=0, d \theta^{j}(v, w)=0
$$

These are independent (check yourself!), so we obtain $c_{1}=4$ and $c_{0}+c_{1}=6$.

\section{Determination of $\operatorname{codim} \mathcal{V}_{2}$.}

Let $\mathbf{G}(2, T \Sigma)$ have local coordinates $\left(x^{i}, u^{a}, p_{i}^{a} ; b_{a}^{i}, c_{a}^{i}, e^{a}, f^{a}\right)$ where the first set gives coordinates for the base point and the second for the plane $v \wedge w$ where

$$
\begin{aligned}
v & =\frac{\partial}{\partial x^{1}}+b_{a}^{i} \frac{\partial}{\partial p_{i}^{a}}+e^{a} \frac{\partial}{\partial u^{a}} \\
w & =\frac{\partial}{\partial x^{2}}+c_{a}^{i} \frac{\partial}{\partial p_{i}^{a}}+f^{a} \frac{\partial}{\partial u^{a}}
\end{aligned}
$$

We have the following conditions and consequences:

$$
\begin{array}{ccc}
\theta^{1}(v)=0 & \Longrightarrow & b^{1}=p_{1}^{1} \\
\theta^{2}(v)=0 & \Longrightarrow & b^{2}=p_{1}^{2} \\
\theta^{1}(w)=0 & \Longrightarrow & c^{1}=p_{2}^{1} \\
\theta^{2}(w)=0 & \Longrightarrow & c^{2}=p_{2}^{2} \\
d \theta^{1}(v \wedge w)=0 & \Longrightarrow & c_{1}^{1}-b_{2}^{1}=0 \\
d \theta^{2}(v \wedge w)=0 & \Longrightarrow & c_{1}^{2}-b_{2}^{2}=0
\end{array}
$$

These six equations are independent and we conclude codim $\mathcal{V}_{2}(\mathcal{I})=6$ and Cartan's test succeeds. Moreover integral manifolds "depend on two functions of two variables" which in this case we see explicitly, as we knew the solutions all along. 
Example 1: The Cauchy-Riemann equations $u_{x^{1}}^{1}=u_{x_{2}}^{2}, u_{x^{2}}^{1}=-u_{x_{1}}^{2}$.

This example is the same as above, except that we now restrict to the submanifold $\Sigma^{\prime} \subset \Sigma$ where $p_{1}^{1}=p_{2}^{2}$ and $p_{2}^{1}=-p_{1}^{2}$. We still have $c_{0}=2$ as $\theta^{1}, \theta^{2}$ remain linearly independent when restricted to $\Sigma^{\prime}$ but we now have $c_{1}=2$ (exercise be sure to express the initial $v$ in terms of 6 variables (e.g., eliminate $\left.p_{1}^{2}, p_{2}^{2}\right)$ ). Similarly, only four of the six equations for $\mathcal{V}_{2}$ remain independent. So here we have the equality $\operatorname{codim}_{E} \mathcal{V}_{2}=4=c_{0}+c_{1}=2+2$.

Here Cartan's test indicates that integral manifolds should depend on two functions of one variable, which we also know to be the case as a (sufficiently generic) real analytic arc uniquely determines a holomorphic map $\mathbb{C} \rightarrow \mathbb{C}$.

Remark 3. Note that in both the above calculations, the calculation of codim $\mathcal{V}_{2}(\mathcal{I})_{p}$ was linear. There is a large class of EDS, called linear Pfaffian systems which are systems defined by one-forms for which this linearity holds. For such systems, there is a simplified version of Cartan's test. Any system of partial differential equations expressed as the pullback of the contact system on the space of jets is a linear Pfaffian system, see, e.g., [13], example 5.1.4.

\section{Example 2: Lagrangian submanifolds.}

Let $\omega$ be the standard symplectic form on $\mathbb{R}^{2 n}$ :

$$
\omega=d x^{1} \wedge d y^{1}+\ldots+d x^{n} \wedge d y^{n} .
$$

An $n$-dimensional submanifold is Lagrangian if it is an integral manifold of $\mathcal{I}=$ $\{\omega\}_{\text {diff }}$.

Given $(p, E) \in \mathcal{V}_{n}(\mathcal{I})$, we can make a linear change of coordinates (while keeping the form of $\omega$ ) so that $E$ is annihilated by $d y^{1}, \ldots, d y^{n}$. This is because the subgroup of $G L\left(T_{p} \mathbb{R}^{2 n}\right)$ leaving $\omega$ invariant is the symplectic group which acts transitively not only on Lagrangian $n$-planes but on all flags within them. Thus all $n$-planes at all points are equivalent and genericity issues don't enter. Any nearby integral $n$-planes at $p$ are given by $d y^{j}=\sum_{k} s^{j k} d x^{j}$ for $s^{j k}=s^{k j}$. Therefore, $\operatorname{dim}\left(\mathcal{V}_{n}(\mathcal{I})_{p}\right)=\left(\begin{array}{c}n+1 \\ 2\end{array}\right)$,

$$
\operatorname{codim}_{E}\left(\mathcal{V}_{n}(\mathcal{I})_{p}, G\left(n, T_{p} \mathbb{R}^{2 n}\right)\right)=\operatorname{codim}_{E}\left(\mathcal{V}_{n}(\mathcal{I}), \mathbf{G}\left(n, T \mathbb{R}^{2 n}\right)\right)=\left(\begin{array}{l}
n \\
2
\end{array}\right),
$$

independent of $p$ and $E$.

Let $e_{1}, \ldots, e_{n} \in E$ be dual to $d x^{1}, \ldots, d x^{n}$ and we use $e_{1}, \ldots, e_{n}$ to build our flag in $E$, i.e., $E_{j}=\left\langle e_{1}, \ldots, e_{j}\right\rangle$. (By the remark above, there are no genericity issues to be concerned with.) 
It is easy to calculate that for $j \leq n$,

$$
H\left(E_{j}\right)=\left\{v \in T_{p} \mathbb{R}^{2 n} \mid d y^{k}(v)=0 \forall k \leq j\right\}
$$

so $c_{j}=j$ for $j \leq n-1$. Since $c_{1}+c_{2}+\ldots+c_{n-1}=\left(\begin{array}{l}n \\ 2\end{array}\right)$, we have involutivity, and integral manifolds depend on 1 function of $n$ variables. (In fact, they can be explicitly constructed by setting $y^{j}=\partial f / \partial x^{j}$ for $f$ an arbitrary function of $x^{1}, \ldots, x^{n}$.)

\section{Periodic billiard orbits}

We now return to the problem of finding $n$-periodic billiard orbits. (The results of this section and the next are joint with Baryshnikov and Zharnitsky.) We have the EDS

$$
\mathcal{I}=\left\{\psi_{i}\right\}_{\text {diff }}
$$

and several independence conditions: that each $\eta^{i} \wedge \eta^{i+1}$ is nonvanishing on an integral manifold. Fortunately we can reduce to a single independence condition thanks to the following lemma:

Lemma 1. It is sufficient to work with the independence condition $\eta^{1} \wedge \eta^{2}$ (or any $\left.\eta^{i} \wedge \eta^{i+1}\right)$.

Proof: Let $X_{i}$ be a dual basis to $\eta^{j}$ of ker $\mathcal{I}_{1}$. Take local coordinates $p_{1}^{\alpha}, p_{2}^{\alpha}$ about $\left[X_{1} \wedge X_{2}\right]$ where we write $[v \wedge w]$ as a nearby point with $v=X_{1}+p_{1}^{\alpha} X_{\alpha}$, $w=X_{2}+p_{2}^{\alpha} X_{\alpha}$.

Introduce the notations $a_{j}=\frac{\cos \left(\alpha_{\mathrm{j}+1}\right)}{2 l_{j}}, b_{j}=\frac{\cos \left(\alpha_{\mathrm{j}-1}\right)}{2 l_{j-1}}$ where we use the notation of $\S 4$. One calculates (see [7]) that

$$
d \psi^{j} \equiv\left(a_{j} \eta^{j+1}+b_{j} \eta^{j-1}\right) \wedge \eta^{j} \bmod I
$$

Moreover $p \in \Sigma$ implies that none of the $a_{j}, b_{j}$ are zero at $p$.

Evaluating the $d \psi^{i}$ at $v \wedge w$ (that is, evaluating at an arbitrary point in our chart) we obtain the $n$ equations on the $p_{j}^{\alpha}$

$$
\begin{array}{ccc}
0 & = & a_{1}+b_{1} p_{2}^{n} \\
0 & = & p_{1}^{3} a_{2}+b_{2} \\
0= & \left(p_{2}^{4} p_{1}^{3}-p_{2}^{3} p_{1}^{4}\right) a_{3}+p_{1}^{3} b_{3} \\
0= & \left(p_{2}^{5} p_{1}^{4}-p_{1}^{5} p_{2}^{4}\right) a_{4}+\left(p_{2}^{4} p_{1}^{3}-p_{2}^{3} p_{1}^{4}\right) b_{4} \\
& \vdots & \\
0 & = & p_{2}^{n} a_{n}+\left(p_{2}^{n} p_{1}^{n-1}-p_{2}^{n-1} p_{1}^{n}\right) b_{n}
\end{array}
$$

of which $n-1$ are independent.

The first equation implies $p_{2}^{n} \neq 0$, which implies that on an integral element on which $\eta^{1} \wedge \eta^{2} \neq 0$, we also have $\eta^{n} \wedge \eta^{1} \neq 0$. The second equation implies 
$p_{1}^{3} \neq 0$ which implies that similarly $\eta^{2} \wedge \eta^{3} \neq 0$. The third equation implies that $\left(p_{2}^{4} p_{1}^{3}-p_{2}^{3} p_{1}^{4}\right) \neq 0$ but this is exactly the condition that $\eta^{3} \wedge \eta^{4} \neq 0$. Continuing, we see that $\eta^{1} \wedge \eta^{2} \neq 0$ implies that all $\eta^{j} \wedge \eta^{j+1} \neq 0$ on an integral element.

Remark 4. Had we instead taken, e.g., $\eta^{1} \wedge \eta^{3}$ as independence condition, (assuming $n>3$ ) we could not have drawn a similar conclusion, see [7].

Introduce notation $\Delta_{j-1}=\left(p_{1}^{j} p_{2}^{j-1}-p_{2}^{j} p_{1}^{j-1}\right)$ with the convention that $p_{1}^{1}=p_{2}^{2}=$ $1, p_{1}^{2}=p_{2}^{1}=0$, so $\Delta_{1}=1$. Then our equations (8) become

$$
a_{j} \Delta_{j-1}+b_{j} \Delta_{j}=0
$$

which we may write in matrix form:

$$
\left(\begin{array}{cccccc}
0 & 0 & 0 & \ldots & 0 & a_{1} \\
a_{2} & b_{2} & 0 & \ldots & 0 & 0 \\
0 & a_{3} & b_{3} & \ldots & 0 & 0 \\
\vdots & \vdots & \vdots & \vdots & \vdots & \vdots \\
0 & 0 & 0 & \ldots & a_{n-1} & b_{n-1}
\end{array}\right)\left(\begin{array}{c}
\Delta_{2} \\
\Delta_{3} \\
\Delta_{4} \\
\vdots \\
\Delta_{n}
\end{array}\right)=\left(\begin{array}{c}
-b_{1} \\
-a_{2} \\
0 \\
\vdots \\
0
\end{array}\right)
$$

and since the $a_{j}$ are nonzero, there is a unique solution for $\Delta_{2}, \ldots, \Delta_{n}$. Now $\Delta_{2}=p_{1}^{3}$ and $\Delta_{n}=p_{2}^{n}$ so $p_{1}^{3}, p_{2}^{n}$ are fixed and the remaining equations on the $p_{i}^{\alpha}$ are independent. In fact one can solve explicitly for all the remaining $p_{1}^{\alpha}, p_{2}^{\alpha}$ in terms of $p_{1}^{4}, p_{2}^{3}, p_{1}^{5}, p_{1}^{6}, \ldots, p_{1}^{n-1}$. Thus the space of integral elements satisfying the genericity condition is of dimension $n-3$.

Proposition 4. The system $\left(\mathcal{I}, \eta^{1} \wedge \eta^{2}\right)$ has codim $\mathcal{V}_{2}(\mathcal{I})=3 n-1, c_{0}=n$, $c_{1}=2 n-2$ and thus fails Cartan's test by one.

Proof: Here $c_{0}$ is just the codimension of the space of one-dimensional integral elements at a point of $\Sigma$. To calculate $c_{1}$, one needs a sufficiently generic vector, $Z=X_{1}+\cdots+X_{n}$ will do. One then sees that $Z$ is contained in a unique twodimensional integral element.

If one ignores the genericity conditions, as $n$ increases the dimensions of integral manifolds can be arbitrarily large (see [7]). The next proposition states that with the genericity conditions, this fails even at the infinitesimal level.

Proposition 5. For all $n$, there are no 3-dimensional integral elements to $\mathcal{I}$ satisfying the genericity conditions.

Proof: On a three dimensional integral element, we must have say $\eta^{1}, \eta^{2}, f_{a} \eta^{a}$ independent where $3 \leq a \leq n$ and the $f_{a}$ 's are some constants. First note that $f_{3}, f_{n}$ must be zero by considering $d \psi^{2}, d \psi^{1}$ respectively. But we also must have 
$\eta^{2}, \eta^{3}$ independent, and since $f_{3}=0$, this implies $\eta^{2}, \eta^{3}, f_{a} \eta^{a}$ must be independent, which, using $d \psi^{3}$ implies that $f_{4}=0$. Continuing in this fashion one obtains that all the $f_{a}$ must be zero.

\section{Three periodic billiard orbits}

Here the space of integral elements satisfying the billiard conditions is a single point. Taking $\eta^{1} \wedge \eta^{3}$ as our independence condition, writing $c_{j}=\cos \left(\alpha_{\mathrm{j}}\right), s_{j}=$ $\sin \left(\alpha_{\mathrm{j}}\right)$, we see that on integral elements

$$
\eta^{2}+\frac{c_{1} l_{2}}{c_{2} l_{3}} \eta^{1}+\frac{c_{3} l_{1}}{c_{2} l_{3}} \eta^{3}=0
$$

Adding this form to the ideal and taking its derivative, we see

$$
\begin{aligned}
& d\left(\eta^{2}+\frac{c_{1} l_{2}}{c_{2} l_{3}} \eta^{1}+\frac{c_{3} l_{1}}{c_{2} l_{3}} \eta^{3}\right) \\
& \quad \equiv\left[\left(-s_{3} c_{1} c_{2}+c_{3} s_{2} c_{1}+c_{3} s_{1} c_{2}\right) l_{1}+\left(-c_{3} s_{1} c_{2}+s_{3} c_{1} c_{2}+c_{3} s_{2} c_{1}\right) l_{2}\right. \\
& \left.\quad+\left(-c_{3} s_{2} c_{1}+s_{3} c_{1} c_{2}+c_{3} s_{1} c_{2}\right) l_{3}\right] \frac{\eta^{1} \wedge \eta^{3}}{c_{2}^{2} l_{3}^{2}}
\end{aligned}
$$

Thus $\mathcal{V}_{1}(\mathcal{I})_{x}=\emptyset$ for general $x \in \Sigma$ and we must restrict to the subvariety of $\Sigma$ where

$$
\begin{aligned}
& \left(-s_{3} c_{1} c_{2}+c_{3} s_{2} c_{1}+c_{3} s_{1} c_{2}\right) l_{1}+\left(-c_{3} s_{1} c_{2}+s_{3} c_{1} c_{2}+c_{3} s_{2} c_{1}\right) l_{2} \\
& +\left(-c_{3} s_{2} c_{1}+s_{3} c_{1} c_{2}+c_{3} s_{1} c_{2}\right) l_{3}=0 .
\end{aligned}
$$

Now recall that a triangle is uniquely determined, e.g., by two of its three angles and the length of one of its sides, we may write

$$
\begin{aligned}
\alpha_{3} & =\frac{\pi}{2}-\alpha_{1}-\alpha_{2} \\
l_{3} & =\frac{l_{1} \sin \left(2 \alpha_{2}\right)}{\sin \left(\pi-2 \alpha_{1}-2 \alpha_{2}\right)} \\
l_{2} & =\frac{l_{1} \sin \left(2 \alpha_{1}\right)}{\sin \left(\pi-2 \alpha_{1}-2 \alpha_{2}\right)}
\end{aligned}
$$

and substituting in, we obtain the equation

$$
6 l_{1} c_{1} c_{2} s_{1} s_{2}=0
$$

which cannot occur on $\Sigma$. 


\section{A few successes of the Cartan-Kähler theorem}

\subsection{The Cartan-Janet theorem}

Given an analytic Riemannian manifold $\left(M^{n}, g\right)$, does there exist a local isometric immersion into Euclidean space $\mathbb{E}^{n+s}$ ? The Cartan-Janet theorem states that for any analytic metric the answer is yes as long as $s \geq\left(\begin{array}{l}n \\ 2\end{array}\right)$. If the metric is special one can sometimes do much better, see $[8,1,6]$ for the cases of space forms and generalizations.

\subsection{Manifolds with exceptional holonomy}

Using EDS Bryant [4] showed that there exist non-symmetric Riemannian manifolds with holonomy $G_{2}$ and $\operatorname{Spin}_{7}$, settling the last open local existence questions in the Riemannian case of Berger's 1953 thesis [2].

\subsection{Existence of calibrated submanifolds}

The abundance of special Lagrangian and other calibrated submanifolds was first proved by Harvey and Lawson [12] using the Cartan-Kähler theorem.

While describing the first two examples would involve too many definitions, we will explicitly describe two cases of applying the Cartan-Kähler theorem to prove existence of calibrated submanifolds.

Definition 6. A calibration on an oriented Riemannian manifold $\Sigma$ is a closed differential form $\phi \in \Omega^{k}(\Sigma)$ such that for all unit volume $(p, E) \in \mathbf{G}(k, T \Sigma)$, $\phi(E) \leq 1$.

There are many variants on the definition. Calibrations are a tool for finding volume minimizing submanifolds of $\Sigma$ because the fundamental lemma of calibrations says that if $i: M \rightarrow \Sigma$ is an immersed submanifold on which $i^{*}(\phi)=\operatorname{vol}_{M}$ then $M$ is volume minimizing in its homology class (assuming $M$ is compact, there are variations when $M$ is noncompact), see [12].

Recently calibrated manifolds have become of central importance because of applications to physics. See, e.g., Joyce's lectures in [15]. Calibrations may be thought of as generalizations of normalized powers of the Kähler form, which itself gives rise to an involutive system (the Cauchy-Riemann equations!). We will discuss two additional calibrations, the special Lagrangian calibration and the associative calibration.

Sometimes a calibration $\alpha$ has a complementary form $\alpha_{c}$ such that

$$
|\alpha(E)|^{2}+\left|\alpha_{c}(E)\right|^{2}=1
$$


for all unit volume planes $E$. In such cases we may define an EDS whose integral manifolds are the submanifolds calibrated by $\alpha$ by taking $\mathcal{I}=\left\{\alpha_{c}\right\}_{\text {diff } f}$.

Example 2 (Special Lagrangian manifolds). On $\mathbb{R}^{2 n}=\mathbb{C}^{n}$ (or any Kähler manifold), consider the differential $n$-form

$$
\alpha=\operatorname{Re}\left(d z^{1} \wedge \cdots \wedge d z^{n}\right),
$$

where $z^{j}=d x^{j}+i d y^{j}$, called the special Lagrangian calibration.

In the special Lagrangian case, a variant of (10) holds. If we take

$$
\alpha_{c}=\operatorname{Im}\left(d z^{1} \wedge \cdots \wedge d z^{n}\right)
$$

then, restricted to Lagrangian n-planes, (10) holds. Moreover, it is easy to see any submanifold calibrated by $\alpha$ is Lagrangian, so $\mathcal{I}=\left\{\omega, \alpha_{c}\right\}_{\text {diff }}$ is an EDS whose integral manifolds are the special Lagrangian submanifolds.

Given $E \in \mathcal{V}_{n}(\mathcal{I})$, we can change coordinates so that $E$ is annihilated by $d y^{1}, \ldots, d y^{n}$. (This is because the system is $S U(n)$ invariant and $S U(n)$ acts transitively on the special Lagrangian planes at a point and even transitively on flags in special Lagrangian planes.) Taking $e_{1}, \ldots, e_{n} \in E$ to be dual to $d x^{1}, \ldots, d x^{n}$, we have $c_{j}=j$ for $1 \leq j \leq n-2$ as in example 2 of $\S 8$. However,

$$
\left.\begin{array}{rl}
\omega & \equiv d x^{n-1} \wedge d y^{n-1}+d x^{n} \wedge d y^{n} \\
\alpha_{c} & \equiv d x^{1} \wedge \cdots d x^{n-2} \wedge\left(d x^{n-1} \wedge d y^{n}-d x^{n} \wedge d y^{n-1}\right)
\end{array}\right\} \bmod d y^{1}, \ldots, d y^{n-2}
$$

shows that $c_{n-1}=n$. The requirement that $\left.\alpha_{c}\right|_{E}=0$ is one additional equation $\left(\sum_{j} s^{j j}=0\right)$ on the set of Lagrangian $n$-planes so the codimension of $\mathcal{V}_{n}(\mathcal{I})$ is one greater than the Lagrangian case and the system is involutive, with solutions depending on two functions of $n-1$ variables.

Example 3 (Associative submanifolds). The 14-dimensional compact Lie group $G_{2}$ arises as the automorphism group of the normed algebra $\mathbb{O}$ of octonions (see e.g., [13] §A.5)), and leaves invariant a 3-form $\phi$ on $\mathbb{R}^{7}=\operatorname{Im} \mathbb{O}$, where $\phi(x, y, z)=$ $\langle x, y z\rangle$. (Here $\langle\cdot, \cdot\rangle$ is the inner-product induced from the norm.) This $\phi$ is a calibration on $\mathbb{R}^{7}$, and it admits a complement as in (10): $\phi_{c}=\frac{1}{2} \operatorname{lm}((x y) z-(z y) x)$. We define an EDS $\mathcal{I}$ for associative submanifolds by taking the components of the $\operatorname{Im} \mathbb{O}$-valued 3-form $\phi_{c}$ as generators. (Since $\phi_{c}$ is constant-coefficient, all of these generators are closed.)

Let $E \in \mathcal{V}_{3}(\mathcal{I})$. Then the stabilizer of $E$ in $G_{2}$ is six-dimensional. Since $G_{2}$ acts transitively on the space of 3 -dimensional integral elements, we conclude

$$
\operatorname{codim}\left(\mathcal{V}_{3}(\mathcal{I})_{p}, G\left(3, T_{p} \operatorname{Im} \mathbb{O}\right)\right)=12-8=4
$$

On the other hand, for any flag in $E, c_{0}=c_{1}=0$ and $c_{2}=4$ (two independent vectors in $E$ determine the third one by multiplication). Thus $\mathcal{I}$ is involutive at $E$ 
(hence involutive everywhere, by homogeneity). Integral manifolds depend on 4 functions of two variables.

\section{Acknowledgements}

These notes are bases on series of lectures I gave at KIAS 2005 and the seventh international conference on geometry, integrability and quantization (respectively March and June 2005). It is a pleasure to thank my Korean and Bulgarian hosts for these lecture series, especially Sung-Eun Koh and Ivalio Mladenov. I also thank T. Ivey, O. Yampolski and V. Zharnitsky for useful comments. Supported by NSF grant DMS-0305829

\section{Bibliography}

\section{References}

[1] E. Berger, R. Bryant, P. Griffiths, The Gauss equations and rigidity of isometric embeddings, Duke Math. J. 50 (1983) 803-892.

[2] M. Berger, Sur les groupes d'holonomie des variÃl'tãl's riemanniennes. (French) C. R. Acad. Sci. Paris 237, (1953). 472-473

[3] G. Birkhoff, Dynamical Systems, Amer. Math. Soc., Providence, RI, 1927.

[4] R. Bryant, Metrics with exceptional holonomy, Ann. of Math. 126 (1987), 525-576.

[5] R. Bryant, S.-S. Chern, R.B. Gardner, H. Goldschmidt, P. Griffiths, Exterior Differential Systems, MSRI Publications, Springer, 1990.

[6] R. Bryant, P. Griffiths,D. Yang, Characteristics and existence of isometric embeddings. Duke Math. J. 50 (1983), no. 4, 893-994.

[7] Y. Baryishnikov, J. Landsberg, V. Zharnitsky, Periodic orbits in Birkoff billiards, in preparation.

[8] E. Cartan, Sur les variétés de courbure constante d'un espace euclidien ou non euclidien, Bull. Soc. Math France 47 (1919) 125-160 and 48 (1920), 132-208; see also pp. 321-432 in Oeuvres Complètes Part 3, Gauthier-Villars, 1955.

[9] —, Sur la théorie des systèmes en involution et ses applications à la relativité, Bull. Soc. Math. Fr. 59 (1931), 88-118; see also pp. 1199-1230 in Oeuvres Complètes, Part 2.

[10] —, Les Systèmes Extérieurs et leurs Applications Géométriques, Hermann, 1945.

[11] V. Guillemin, The Radon transform on Zoll surfaces. Advances in Math. 22 (1976), no. $1,85-119$.

[12] F. Harvey, H. Lawson, Calibrated geometries, Acta Math. 148 (1982), 47-157.

[13] T. Ivey, J. Landsberg, Cartan for beginners: differential geometry via moving frames and exterior differential systems. Graduate Studies in Mathematics, 61. American Mathematical Society, Providence, RI, 2003. xiv+378 pp. 
[14] V. Ivrii, The second term of the spectral asymptotics for the Laplace-Beltrami operator on manifolds with boundary, Functsional. Anal. i Prilozhen., 14, (1980), N 2, 25-34.

[15] M. Gross, D. Huybrechts, D. Joyce Calabi-Yau manifolds and related geometries. Lectures from the Summer School held in Nordfjordeid, June 2001. Universitext. Springer-Verlag, Berlin, 2003. viii+239 pp.

[16] J. Landsberg Exterior differential systems: A geometric approach to PDE in Topology and geometry, proceedings of workshop in pure math, vol. 17 part III, Korean academic council (1998). pp. 77-100.

[17] M. Rychlik, Periodic points of the billiard ball map in a convex domain, J. Diff. Geometry 30 (1989) 191-205.

[18] L. Stojanov, Note on periodic points of the billiards, J. Diff. Geometry 34 (1991) 835-837.

[19] H. Weyl, Über die saymptotische Verteilung der Eigenwerte, Göttinger. Nachr. 110117 (1911).

[20] M. Wojtkovski, Two applications of Jacobi fields to the billiard ball problem, J. Diff. Geometry 40 (1994) 155-164. 